\title{
Diverticiencia: Experiencia de enseñanza-aprendizaje de las ciencias entre niveles educativos
}

Ana Aragüés Díaz - IES Valle del Ebro

Beatriz Carrasquer Álvarez - Universidad de Zaragoza
0000-0002-8228-0813

0000-0001-9336-4644

Recepción: 05.05.2020 | Aceptado: 30.11.2020

Correspondencia a través de ORCID: Ana Aragüés Díaz

iD 0000-0002-8228-0813

Citar: Aragües, A. y Carrasquer, B. (2020). Diverticiencia: Experiencia de enseñanza-aprendizaje de las ciencias entre niveles educativos. REIDOCREA, 9, 244-251.

Resumen: En este estudio se muestran diversas experiencias de divulgación científica llevadas a cabo por alumnado de segundo de bachillerato para enseñar ciencias a estudiantes de segundo de Educación Secundaria Obligatoria. El primer objetivo es que el alumnado de último curso de Bachillerato desarrolle capacidades de organización y colaboración, así como la que aprendan a transmitir conocimientos en público de forma clara, poniendo en práctica contenidos transversales y específicos, incluyendo los relacionados con la actividad científica. Además, se quiere fomentar el interés del alumnado por las ciencias desde edades tempranas. Tras la experiencia los docentes de secundaria pasaron un cuestionario al alumnado de ESO para recoger el grado de implicación, interés y adquisición de contenidos. Las respuestas recogidas corroboraron el alto grado de implicación, desarrollo de habilidades la adquisición del contenido observado en los estudiantes de $2^{\circ}$ de Bachiller. Así mismo el alumnado de secundaria ha demostrado haber adquirido los contenidos y competencias deseados. Se pone de manifiesto que la implementación de experiencias indagativas mediante cooperación entre niveles educativos ha resultado ser una herramienta útil para conseguir los objetivos perseguidos.

Palabra clave: Cooperación Educativa

Fun: Science teaching-learning experience between educational levels

Abstract: This work shows various experiences of scientific dissemination carried out by last-year high school students (pre-university students) to teach sciences to second-year students of Compulsory Secondary Education. The first goal is to develop organizational and cooperation capacities of the students of the last year of high school as well as the opportunity for them to acquire capacities to transmit knowledge in a clear way. Thus, they have to put into practice transversal and specific contents, and also those related to scientific activity. It is also proposed to promote the interest of compulsory secondary education students in science, from young ages. After the experience, the secondary school teachers given a questionary to the students of second year of Compulsory Secondary to collect and evaluate their degree of involvement, interest and content acquisition. The high degree of involvement, development of skills and acquisition of the desired content showed by last-year students at all stages of the experience was corroborated by second-year students answers. They also acquired the desired contents and competencies. This shows that the implementation of inquiry-based experiences by cooperation between educational levels has been a useful tool to achieve the objectives pursued.

Keyword: Educational Cooperation

\section{Introducción}

Los resultados obtenidos en el último informe del Programa para la Evaluación Internacional de Estudiantes (Programme for International Student Assessment, PISA) promovido por la OCDE, no son muy alentadores para las ciencias y las matemáticas, de acuerdo información difundida por el Gobierno de España (Instituto Nacional de Evaluación Educativa, www.educacionyfp.gob.es). Este programa intenta delimitar, describir y explicar lo que los jóvenes de 15 años conocen y saben hacer al final de su etapa educativa obligatoria, aplicando sus conocimientos a una variedad de entornos y contextos, dentro y fuera de su entorno escolar. El estudio analiza principalmente tres competencias: lectora, matemática y científica. En concreto, la competencia científica incluye el discurso razonado sobre ciencia y tecnología para explicar fenómenos 
científicos, valorar y diseñar investigaciones científicas, e interpretar datos y pruebas científicas. De acuerdo con los resultados recogidos mediante el mencionado programa (Instituto Nacional de Evaluación Educativa), el 21\% de los estudiantes de España se encuentra en un nivel en el cual suelen necesitar apoyo para abordar cuestiones relacionadas con la ciencia. Solo un $4,2 \%$ está suficientemente capacitados y conocen lo suficiente de las ciencias como para poder aplicar creativa y autónomamente sus conocimientos y habilidades a una amplia variedad de situaciones, incluyendo aquellas que les resultan desconocidas, porcentaje significativamente inferior a la media de los países de la OCDE $(6,8 \%)$ y del Total UE $(6,9 \%)$. Es por ello que, en línea con diversas investigaciones (Jiménez y Llitjós 2006, Pujolás 2012, Méndez 2015) se considera necesario que el alumnado adquiera una visión favorable hacia las ciencias, desarrollando competencias y aprendiendo contenidos de manera colaborativa, lúdica, y cercana a la realidad mediante fenómenos cotidianos. Este tipo de método de enseñanza-aprendizaje fomenta además la motivación y la inclusión de los estudiantes en relación al aprendizaje de materias científicas (Tortosa y Gorchs 2011, Pujolás 2012, Méndez 2015).

\section{Objetivo y contexto}

Teniendo en cuenta lo anteriormente expuesto, desde hace varios años, en el Instituto de Educación Secundaria donde se desarrolla el estudio, todos los meses de marzo, se lleva a cabo la Semana de la Ciencia. En esos días se realizan talleres científicos, mostración de experimentos o exposiciones que el propio alumnado ha realizado así como visitas guiadas. Los estudiantes pueden entre otras actividades, observar una gran tabla periódica así como maquetas de modelos atómicos elaborados por alumnado de ESO, exposiciones como "Un Universo de luz" que hace un interesante reflexión sobre la importancia de la luz en nuestras vidas, y participar en talleres de "Biología forense para principiantes" organizado por la Universidad de Navarra, o de análisis aguas, y en charlas como la llevada a cabo por José Basilio Galván que abordan el concepto de desorden del Universo y Entropía, entre otras. En este contexto se implementa también la experiencia que se presenta en este trabajo, que comenzó en el 2011-12, y que, de manera continuada se ha ido celebrando cada curso. En ella, el alumnado de segundo de bachiller organiza una serie de talleres y enseña ciencias al alumnado de segundo de ESO.

A nivel Europeo, se insta en las últimas décadas a que los encargados de las tomas de decisiones exijan a los organismos responsables de aplicar cambios en el sistema educativo a nivel, local, nacional, y de la Unión europea, que tomen medidas por la mejora enseñanza de las ciencias (Rocard 2007). Se apuesta por nuevas formas de pedagogía que deberían promover y apoyar activamente enfoques hacia la investigación en las escuelas. Le educación científica debería ser un componente esencial de proceso de aprendizaje continuo para toda la ciudadanía, en todos los niveles educativos (Hazelkorn 2015). Es decir, considerar la ciencia como componente fundamental de la educación obligatoria. Y adoptar un enfoque de investigación de la educación científica. Este enfoque debe fundamentarse en llevar la autenticidad de la práctica científica al aula, y motivar e involucrar a los alumnos, fomentando la educación basada en metodologías de indagación, Inquiry-Based Science Education (DomènechCasal 2019, Moraga, Espinet y Merino 2019, Couso, Jímenez-Liso, Refojo y Sacristán 2020).

De acuerdo con Méndez (2015), el alumnado de secundaria en España obtiene bajos resultados de aprendizaje en ciencias debido en gran medida a su desmotivación. Y el 
aprendizaje cooperativo provoca un cambio motivacional altamente positivo comparado con el aprendizaje tradicional (Méndez 2013, Méndez 2015). En este contexto, no cabe duda de que los talleres de ciencia son una herramienta muy eficaz para acercar el trabajo científico al alumnado (Carretero y Sánchez-Guadix 2008), que va a poder comprobar que los fenómenos cotidianos que nos rodean tienen una explicación, pudiendo encontrar, razonar y exponer sus conclusiones por ellos mismos. Solo cuando los fenómenos que nos rodean, tales como las reacciones químicas, se ven en la práctica, es cuando adquieren un significado y llegan a comprenderse. El alumnado es protagonista de su propio proceso de enseñanza aprendizaje y de su alfabetización científica. Además, los talleres despiertan el interés por determinadas asignaturas que gran parte del alumnado considera complicadas e inaccesibles. Este tipo de propuestas son agradables, divertidas y cooperativas, ayudando a la adquisición de contenidos científicos, y a que éstos sean adquiridos de manera significativa. Máxime cuando estos talleres son preparados por alumnado de último curso de Bachiller para enseñar a alumnado de primeros cursos de ESO, convirtiéndose en una experiencia entre alumnado de diferentes niveles educativos en la que todos adquieren conocimientos, tal y como se plantea en este trabajo.

\section{Métodos}

\section{Participantes}

La experiencia de los talleres de Diverticiencia comenzó en el curso 2011-12 por alumnado del IES Valle del Ebro. En concreto, durante el curso 2017-18, en el cual se centran los resultados de este trabajo, 12 estudiantes de la asignatura optativa Experiencias de Física y Química de segundo de Bachiller prepararon los talleres por los que pasaron 193 alumnos/as de segundo de ESO.

Como forma de aproximar la ciencia a los más pequeños del centro educativo, los estudiantes de Bachiller, en grupos de tres personas, prepararon curiosos experimentos de electrostática, presión, centro de gravedad, rozamiento, índice de refracción, y algunas reacciones químicas. Para cada una de las experiencias diseñaron un tríptico incluyendo título de la experiencia, materiales (todos ellos cotidianos), marco teórico, procedimiento experimental. Se muestra un ejemplo en la Figura 1.
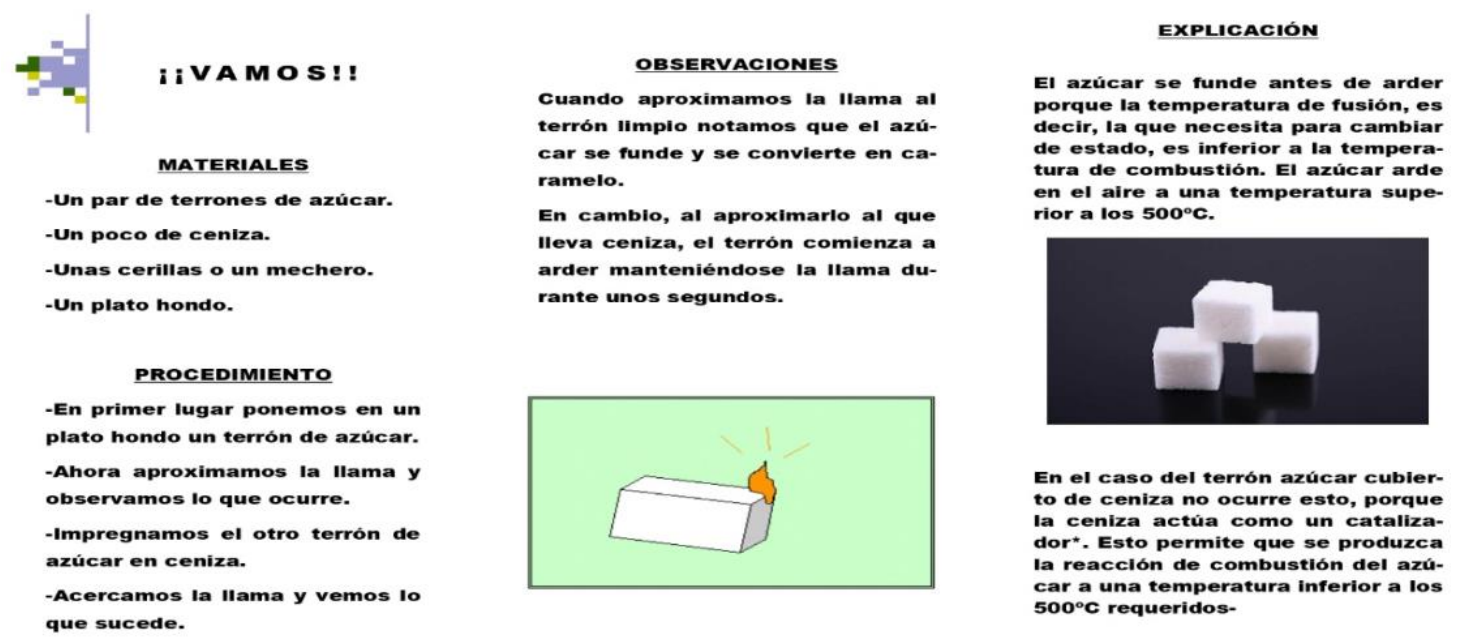

Figura 1. Ejemplo de tríptico elaborado por alumnos de $2^{\circ}$ de Bachillerato titulado ¿Conseguiremos que arda un terrón de azúcar? 
Durante varias sesiones debatieron por grupos y buscaron información acerca de los aspectos a incluir en el tríptico informativo, además llevaron a cabo las experiencias trayendo el material necesario para su desarrollo. En la Tabla 1 se resumen los experimentos desarrollados por el alumnado de Bachiller. Estos experimentos son llevados a cabo en el mes de marzo durante la semana de la ciencia, donde se desarrollan entre otras actividades estos talleres denominados Diverticiencia, y en el que se exponen al alumnado de $2^{\circ}$ de ESO donde participan y son tutorizados por el alumnado de Bachiller en las experiencias programadas.

Tabla 1. Selección de experimentos desarrollados en los talleres Diverticiencia.

\begin{tabular}{ll}
\hline Haribo chisposo & $\begin{array}{l}\text { Observar cómo cambias los reactivos en una reacción química, incluso echando } \\
\text { chispas o convirtiéndose en humo. El punto de fusión del reactivo (osito de la marca } \\
\text { Haribo) es de } 362^{\circ} \mathrm{C} .\end{array}$ \\
\hline La botella succionadora & $\begin{array}{l}\text { Observar la acción de la presión atmosférica con una botella llena de agua, una } \\
\text { gasa y un palillo. }\end{array}$ \\
\hline La lámpara de lava & $\begin{array}{l}\text { Explicar los fenómenos que ocurren con reactivos de distintas densidades } \\
\text { empleando un bote de cristal, agua, aceite, colorante y una pastilla efervescente. }\end{array}$ \\
\hline La cascada de humo & $\begin{array}{l}\text { Observar y explicar cómo el humo dentro de una botella, al ser más denso que el } \\
\text { aire, cae cómo una cascada. }\end{array}$ \\
\hline Pasta de dientes para & $\begin{array}{l}\text { Observar cómo al juntar agua oxigenada con yoduro de potasio se descompone en } \\
\text { agua y oxígeno que en contacto con jabón forma una espuma densa que se }\end{array}$ \\
\hline elefantes & $\begin{array}{l}\text { Obpande. } \\
\text { Serpiente del faraón } \\
\text { bicarbonato sódico y azúcar. Al mezclar ciertas sustancias pueden surgir otras nuevas } \\
\text { con características muy particulares como las del caso de esta combustión. }\end{array}$ \\
\hline Moco loco & $\begin{array}{l}\text { Observar cómo mezclando detergente y cola blanca obtenemos otra sustancia con } \\
\text { propiedades muy distintas como es en este caso una especie de goma viscosa. }\end{array}$ \\
\hline Líquido parece, sólido no & $\begin{array}{l}\text { Observar las propiedades de un líquido no newtoniano creando uno a través de } \\
\text { maicena y agua. Para ello lo tocamos. }\end{array}$ \\
\hline es & Apreciar el elevado calor específico del agua observando que \\
\hline El globo que no explota iris líquido & $\begin{array}{l}\text { Observar cómo sustancias de distintas densidades no se pueden mezclar, obteniendo } \\
\text { como resultado una simulación del espectro de la luz, el arcoíris. }\end{array}$ \\
\hline La jeringa infladora & $\begin{array}{l}\text { Comprobar que se puede inflar dentro de una jeringuilla objetos que ya contienen aire } \\
\text { en su interior. }\end{array}$ \\
\hline La unión no siempre hace & $\begin{array}{l}\text { Observar qué ocurre al poner un globo encima de muchas chinchetas clavadas en } \\
\text { una base de cartón al poner mucho peso encima de él. }\end{array}$ \\
\hline &
\end{tabular}

\section{Instrumentos}

Tras la implementación de los talleres, los docentes de $2^{\circ}$ de ESO pasaron una encuesta-rúbrica al alumnado que participó en los talleres. La encuesta-rúbrica se inspiró en el cuestionario planteado en el estudio de Marbà y Mázquez (2010), así como en los criterios evaluables exigidos a nivel nacional en segundo de ESO y segundo de Bachiller en relación al bloque 1 Actividad Científica de contenidos de Física y Química, en ESO y Bachillerato (Gobierno de España, 2015). Y trataron de incluirse cuestiones relacionadas con conceptos $(C)$, procedimientos $(P)$ y actitudes $(A)$.

Se formularon una serie de afirmaciones que fueron clasificadas en cuatro categorías: Ciencia y diversión, considerando las ciencias como algo lúdico, cercano y sencillo (ítems 1, 5, 9, 13, 17), Interés y curiosidad por la ciencia, incluyendo comprender fenómenos de la vida diaria y el incluir dicha materia en sus perspectivas de futuro formativo y laboral (ítems $3,7,11,15,19$ ), Actuación del alumnado de bachiller (ítems $2,6,10,14,18,21$ ), Ciencia y conocimiento (ítems 4, 8, 12, 20, 22). Fueron valoradas mediante escala liker de 5 puntos (muy en desacuerdo, 1, en desacuerdo, 2, indiferente, 3, de acuerdo, 4, muy de acuerdo, 5). En la Tabla 2 se presentan las afirmaciones planteadas, y se especifica junto con cada una de ellas, el criterio evaluado y el nivel educativo (ESO o Bachiller), así como el tipo de contenidos (C, P, A). Se recogieron los 
datos en hojas Excel para calcular promedio por alumno, ítem, curso. La encuestarúbrica se inspiró en el cuestionario planteado en el estudio de Marbà y Márquez (2010) utilizado en ESO.

Tabla 2. Resumen de la encuesta-rúbrica de evaluación.

\begin{tabular}{|c|c|c|c|c|c|}
\hline ITEM & $\begin{array}{l}\text { CRITERIO } \\
\text { EVALUADO }\end{array}$ & $\begin{array}{c}\text { TIPO DE } \\
\text { CONTENIDO }\end{array}$ & ITEM & $\begin{array}{l}\text { CRITERIO } \\
\text { EVALUADO }\end{array}$ & $\begin{array}{c}\text { TIPO DE } \\
\text { CONTENIDO }\end{array}$ \\
\hline $\begin{array}{l}\text { 1. Me lo he pasado muy } \\
\text { bien probando los } \\
\text { experimentos. }\end{array}$ & $\mathrm{ESO}, 6$ & ( & $\begin{array}{l}\text { 12. He comprendido la } \\
\text { relación entre la } \\
\text { superficie y la presión. }\end{array}$ & $\mathrm{ESO}, 1$ & 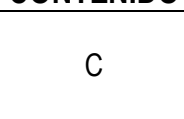 \\
\hline $\begin{array}{l}\text { 2. Las explicaciones } \\
\text { dadas por los } \\
\text { alumnos/as de } 2^{\circ} \text { de } \\
\text { Bachiller han sido } \\
\text { claras. }\end{array}$ & $\begin{array}{c}\text { ESO, } 5 \\
\text { Bach, 1, } 2\end{array}$ & $C, P$ & $\begin{array}{l}\text { 13. No hace falta } \\
\text { mucho para divertirse } \\
\text { con la ciencia. }\end{array}$ & ESO, 4. & A \\
\hline $\begin{array}{l}\text { 3. Probaría estos } \\
\text { experimentos en casa. }\end{array}$ & $\mathrm{ESO}, 4$ & $P, A$ & $\begin{array}{l}\text { 14. Los alumnos/as de } \\
2^{\circ} \text { de Bachiller me han } \\
\text { dejado participar y } \\
\text { ayudar. }\end{array}$ & $\begin{array}{l}\text { ESO, } 6 \\
\text { Bach, } 1\end{array}$ & $P$ \\
\hline $\begin{array}{l}\text { 4. Estos experimentos } \\
\text { me han ayudado a } \\
\text { ampliar mis } \\
\text { conocimientos. }\end{array}$ & ESO, 1 & C & $\begin{array}{l}\text { 15. Estos } \\
\text { experimentos han } \\
\text { hecho que me interese } \\
\text { más por la ciencia. }\end{array}$ & $\mathrm{ESO}, 6$ & A \\
\hline $\begin{array}{l}\text { 5. Me he dado cuenta } \\
\text { que puedo hacer } \\
\text { experimentos con } \\
\text { materiales caseros. }\end{array}$ & ESO, 4 & $P$ & $\begin{array}{l}\text { 16. Me he dado } \\
\text { cuenta de que se } \\
\text { puede hacer un objeto } \\
\text { invisible. }\end{array}$ & $\mathrm{ESO}, 2$ & C \\
\hline $\begin{array}{l}\text { 6. Los alumnos/as de } 2^{0} \\
\text { de Bachiller han sido } \\
\text { simpáticos. }\end{array}$ & ESO, 6 & A & $\begin{array}{l}\text { 17. Me he dado } \\
\text { cuenta de que se } \\
\text { puede hacer ciencia } \\
\text { fuera de la clase } \\
\text { ordinaria. }\end{array}$ & $\mathrm{ESO}, 3$ & $P, A$ \\
\hline $\begin{array}{l}\text { 7. Me gustaría participar } \\
\text { en la semana de la } \\
\text { ciencia años } \\
\text { posteriores. }\end{array}$ & $\mathrm{ESO}, 6$ & A & $\begin{array}{l}\text { 18. Me gustaría ser } \\
\text { como los estudiantes } \\
\text { de } 2^{\circ} \text { de bachillerato } \\
\text { cuando tenga su } \\
\text { edad. }\end{array}$ & $\begin{array}{c}\text { ESO, } 6 \\
\text { Bach, 1, } 2\end{array}$ & $P, A$ \\
\hline $\begin{array}{l}\text { 8. Estos experimentos } \\
\text { me han ayudado a } \\
\text { comprender mejor los } \\
\text { fundamentos científicos. }\end{array}$ & ESO, 1 & C & $\begin{array}{l}\text { 19. Me gustaría } \\
\text { dedicarme a la } \\
\text { ciencia. }\end{array}$ & $\mathrm{ESO}, 6$ & A \\
\hline $\begin{array}{l}\text { 9. Los experimentos me } \\
\text { han parecido divertidos. }\end{array}$ & $\mathrm{ESO}, 6$ & A & $\begin{array}{l}\text { 20. He afianzado los } \\
\text { conceptos teóricos } \\
\text { vistos en clase. }\end{array}$ & ESO, 1, 3 & C \\
\hline $\begin{array}{l}\text { 10. Los folletos } \\
\text { explicaban los } \\
\text { experimentos de forma } \\
\text { clara. }\end{array}$ & $\begin{array}{l}\text { ESO, } 5 . \\
\text { Bach 1,2. }\end{array}$ & $C, P$ & $\begin{array}{l}\text { 21. Los alumnos/as de } \\
\text { bachillerato se han } \\
\text { preocupado de que } \\
\text { me enterara de todo } \\
\text { bien. }\end{array}$ & $\begin{array}{c}\text { ESO, } 5,6 \\
\text { Bach, } 1\end{array}$ & $C, P, A$ \\
\hline $\begin{array}{l}\text { 11. Me gustaría ampliar } \\
\text { información sobre algún } \\
\text { experimento. }\end{array}$ & ESO, 6 . & $\mathrm{C}, \mathrm{A}$ & $\begin{array}{l}\text { 22. He comprobado } \\
\text { que una combustión } \\
\text { requiere oxígeno. }\end{array}$ & ESO, 2 & $C, P$ \\
\hline
\end{tabular}

\section{Resultados}

Es interesante resaltar algunas frases concretas recogidas de las impresiones del alumnado de segundo de ESO durante la implementación de los talleres. Entre ellas destacan:

- Leímos mensajes secretos escritos con tinta invisible

- Aprendimos a meter y sacar un huevo de una botella

- Vimos latas de refrescos saltar al calentarlas con una llamita - o el azúcar que se convirtió en carbón delante de nuestros ojos 
- Vasos de aceite corporal que desaparecían

- Bolsas de té que ascendían en contra de la gravedad

- Vimos una botella fumando, encender una vela a distancia

- Un guante que se mueve solo y una lata de refresco que corría detrás de un globo

- Encender un algodón con papel de aluminio

- La vela que hacía ascender el agua en contra de la gravedad

- Una pila hecha con un limón

- Una caja de quesitos que subía cuesta arriba sin que nadie la empujara

Todos los ítems han sido valorados con una puntuación entre el 3,5 y el 4,5 a excepción del ítem 6: "Los alumnos/as de Bachillerato han sido simpáticos" el cual ha obtenido una puntuación de 4,7, y del valor mínimo 2,9, correspondiente al ítem 19: "Me gustaría dedicarme a la ciencia". La categoría relacionada con 'Ciencia y diversión' ha sido puntuada con un 4,5 sobre 5 de modo que es posible deducir que la actividad ha resultado amena a los estudiantes de $2^{\circ}$ de ESO. La puntuación mínima, 3,62 en cuanto a categoría de items corresponde a la categoría 'Interés por la ciencia'. Las dos categorías restantes 'Actuación' y 'Ciencia y conocimiento' han sobrepasado un 4 (Satisfechos), nuestro objetivo límite.

El grado de implicación y desarrollo de competencias y adquisición de los contenidos deseados observados en el alumnado de Bachillerato ha sido alto en todas las etapas de la experiencia, desde preparación de las experiencias, en la etapa de investigación, hasta evaluación, pasando por la implementación del taller. Esto se ha corroborado con las respuestas dadas por el alumnado de secundaria en la categoría Actuación de la encuesta-rúbrica. Así mismo, el alumnado de ESO ha demostrado haber adquirido los contenidos y competencias deseados en función de las respuestas dadas en las categorías Ciencia y diversión, Ciencia y conocimiento e Interés y curiosidad por la ciencia.

Se pone así de manifiesto que la implementación de experiencias indagativas mediante cooperación entre niveles educativos ha resultado ser una herramienta útil para conseguir los objetivos perseguidos de manera satisfactoria. En concreto, en relación a la adquisición de contenidos conceptuales (durante la preparación de los trípticos informativos de los talleres), procedimentales del alumnado de Bachiller (capacidad de colaboración, organización, transmisión de información en relación a las ciencias, fundamental en el bloque de contenidos de Actividad Científica), y actitudinales en cuanto a que ha fomentado que el alumnado de $2^{\circ}$ de ESO considere las ciencias como algo interesante, lúdico, cercano y sencillo que le ayuda a comprender fenómenos de la vida diaria. Ello queda patente tanto en las respuestas de la encuesta-rúbrica como en las frases recopiladas que se muestran en el apartado de resultados.

Cabe destacar que en función de los resultados, la afirmación relacionada con incluir la ciencia en las perspectivas de futuro laboral del alumnado, ítem 19, obtiene el valor más bajo de 2,9 lo cual tampoco es negativo, teniendo en cuenta que sí lo consideran en sus perspectivas formativas, ítem 18. Por otro lado, de los cuadernos de trabajo de los alumnos de Bachiller de la asignatura de 'Experiencias en Física y Química' se aprecian conclusiones relacionadas con que la experiencia les ha hecho recapacitar acerca de lo que supone un trabajo de investigación

En relación a la auto-evaluación de la experiencia, como aspectos a mejorar cabe destacar la precaución en su desarrollo especialmente en las que por ejemplo se desprende algún tipo de gas como fue con la 'Serpiente del Faraón'. En esta reacción de combustión entre bicarbonato sódico y azúcar produce la liberación de vapor de mercurio y otros gases tóxicos. La realización consecutiva de la práctica a lo largo de la 
jornada puede conllevar a una cierta saturación de los mismos en la atmósfera en caso de no disponer de una campana de extracción en el laboratorio.

\section{Discusión}

El aprendizaje colaborativo entre niveles educativos con enfoque indagativo, lúdico y participativo ha resultado ser una herramienta alternativa al aprendizaje tradicional que ha ayudado a conseguir los objetivos pretendidos.

La colaboración entre el alumnado de $2^{\circ}$ de Bachiller y $2^{\circ}$ de ESO ha reforzado la comunicación y ha ayudado a afianzar conocimientos científicos de diversa índole, no sólo de concepto, sino también de procedimiento y actitud, capacidad de colaboración, organización, transmisión de información e interés por las ciencias. Los estudiantes de ESO han podido aprender ciencia de manera activa y motivadora a través de sencillas actividades. De esta forma el alumnado de ambos cursos se socializa, se responsabiliza y expresa el intercambio de ideas.

A pesar de que la preparación de experiencias prácticas lleva consigo un gran esfuerzo, al compartir esta tarea entre docentes y alumnado de $2^{\circ}$ de Bachiller esta herramienta resulta ser una opción muy recomendable, especialmente para aprender ciencias. La selección, búsqueda de información, planificación, implementación corre a cargo de dicho alumnado, a la que vez que adquiere formación. Además cabe destacar cómo la realización de estas prácticas colaborativas fomenta la cooperación entre niveles educativos, ayudándose entre ellos en el aprendizaje y adquisición de competencias, y fomentando la inclusión del alumnado con mayores dificultades o con menor predisposición a la adquisición de conocimientos de ciencias. Por último, cabe mencionar que el impacto de estos talleres sobre los estudiantes de ESO parece ser especialmente positivo en la medida que una gran mayoría expone querer ser como los estudiantes de Bachillerato cuando tengan su edad, entendiendo que deberán haber elegido una opción científica de Bachiller. Es por tanto, una forma de motivar al alumnado (Carretero y Sánchez 2008) desde edades tempranas en la elección de materias científicas.

\section{Referencias}

Carretero, MB. y Sánchez-Guadix, MA. (2008). Talleres para celebrar la semana de la ciencia. Revista Eureka sobre enseñanza y Divulgación de las Ciencias, 5(1), 62-74.

Couso, D., Jiménez-Liso, MR., Refojo, C. y Sacristán, JA. (coord.) (2020). Enseñando ciencia con ciencia. Madrid: FECYTFundación Lilly.

Domènech-Casal, J. (2019). Retorno a Karlsruhe: una experiencia de indagación con la Tabla Periódica para aprender la estructura y propiedades de los elementos. Revista Eureka de Enseñanza y Divulgación de las Ciencias, 16(1), 1201, 1-18.

Hazelkorn, E. (2015). Science education for responsible citizenship: report to the European Commission of the Expert Group on Science Education. Publications Office of the European Union.

Jiménez, G., Llobera, R. y Llitjós, A. (2006). La atención a la diversidad en las prácticas de laboratorio de química: los niveles de abertura, Enseñanza de las Ciencias, 24(1), 59-70.
Marbà Tallada, A. y Márquez, C. (2010). ¿Qué opinan los estudiantes de las clases de ciencias? Un estudio transversal de sexto de primaria a cuarto de ESO. Enseñanza de las Ciencias, 28(1), 19-30.

Méndez, D. 2015. Estudio de las motivaciones de los estudiantes de secundaria de física y química y la influencia de las metodologías de enseñanza en su interés. Educación XX1, 18(2), 215-235.

Moraga SH., Espinet M. y Merino CG. (2019) El contexto en la enseñanza de la química: Análisis de secuencias de enseñanza y aprendizaje diseñadas por profesores de ciencias en formación inicial. Revista Eureka Sobre Enseñanza y Divulgación de Las Ciencias, 16(1), 1604-1614.

Pujolás, P. (2012). Aulas inclusivas y aprendizaje cooperativo. Educatio siglo XXI: Revista de la Facultad de Educación, 30(1), 89-112.

Rocard, M. (2007). Science education now: A renewed pedagogy for the future of Europe, report to the European Commission of 
the Expert Group on Science Education Office for Official Publications of the European Communities.
Tortosa, M. y Gorchs, R. (2011). Análisis de las prácticas de química de futuros ingenieros: propuestas de mejora efectivas en el marco del espacio europeo de educación superior. Revista Electrónica de Enseñanza de las Ciencias, 10(3), 531-549. 